\title{
Conceptual Development and Content Validation of a Multicultural Instrument to Assess the Normalization of Gender-Based Violence against Women
}

\author{
Maddalena Rodelli ${ }^{1}$ (D) Kleio Koutra ${ }^{2}$ (D) Karen Birna Thorvaldsdottir ${ }^{3}$ (D) \\ Hulya Bilgin ${ }^{4}$ (D) Nikoleta Ratsika ${ }^{2}$. Ines Testoni ${ }^{1}$ (D) . Denise M. Saint Arnault ${ }^{5}$ (D)
}

Accepted: 30 April 2021 / Published online: 7 June 2021

(C) The Author(s) 2021

\begin{abstract}
The normalization of gender-based violence (GBV) consists of all those cultural beliefs and values that sustain, justify, or minimize GBV perpetration. Acknowledging the lack of instruments addressing the normalization of GBV and its constitutive sociocultural dimensions, this article presents the conceptual development and initial validation of the Normalization of gender-based violence against women scale. This 18 -item instrument could be used to assess the normalization of violence against women in GBV survivors of various cultural contexts. The scale has been developed through a sizeable mixed-methods study. This paper reports the qualitative portion of the study that allowed the development of the instrument and assessment of its content and face validity. In particular, the method section details the process by which the assessed scale's domain has been identified through an expert panel workshop, the analysis of GBV survivor's interviews, and the review of existing scales. The assessment of face and content validity, trough expert judges' evaluation and Cognitive Interviewing, is presented. This instrument is the first normalization scale developed by a multicultural team for use with violence survivors. The techniques used to construct this scale aimed to capture cultural aspects of normalization that might be shared across women from diverse groups. Therefore, its use could enable social or health care providers worldwide to program or evaluate the effectiveness of interventions to contrast GBV by promoting a clearer understanding of cultural and social norms that sustain the acceptance and normalization of violence.
\end{abstract}

Keywords Gender-based violence $\cdot$ Normalization - Survivors - Scale development · Content validity $\cdot$ Cognitive interviewing

Maddalena Rodelli

maddalena.rodelli@unipd.it

Extended author information available on the last page of the article 


\section{Introduction}

Gender-based violence (GBV) can be defined as any violent act directed toward individuals basing on socially ascribed gender differences (United Nations, 1993). Although anyone can experience GBV, previous research has shown that women (World Health Organization, 2016) and transgender and gender non-conforming individuals (Wirtz et al., 2020) are the groups who are more disproportionally affected by GBV. In this paper, we focus on GBV against women, which has been indicated as the most widespread violation of human rights, affecting about onethird of all women worldwide (World Health Organization, 2016). We consider of particular interest that, despite such a considerable and persistent prevalence, GBV against women is still a highly underreported phenomenon, whose disclosure is prevented by various factors operating at an individual, sociocultural, and structural level (Gracia, 2004).

A significant, albeit neglected, cultural factor hindering GBV disclosure is constituted by its normalization. Normalization of violence against women consists of globally widespread cultural beliefs and values sustaining and justifying GBV perpetration as a normal component of male-female relationships (Wood, 2001). Foucault (1990) was one of the first scholars to use the term normalization, defining it as the process through which ideas and behaviors that may fall outside of social norms come to be regarded as normal. Since violence usually represents a violation of social norms in contemporary societies, normalization of GBV can be conceptualized as the process through which men's violent behaviors against women end up being presented as natural or normal. In this regard, Butler's significant work (1999) suggests that, in patriarchal societies, femininity is socially constructed and is represented as passive, vulnerable, and submissive, while masculinity is linked with dominance, aggression, and desire. These assumptions contribute to promoting the idea that men's sexual aggression is simply natural, and, therefore, men's violent behaviors against women must be considered somewhat normal (French, 2003; Messerschmidt, 2012; Sinko et al., 2020). Men's violence against women can also be conceived as an instrument to maintain traditional gendered hierarchies (Bloom, 2008). According to Lundgren (2004), abuse in an intimate partnership reinforces normative ideals of masculine domination and female subordination. The experience of abuse strengthens the internalized assumption that violence is a normal part of that subordination.

Previous research refers to the normalization of GBV against women as a process that seems to play an important role both in increasing the incidence of violence (Lundgren, 2004) and decreasing women's ability to recognize, report, escape from, seek help for, or heal from violence (Fugate et al., 2005; Morrison et al., 2006; Overstreet \& Quinn, 2013; Sinko et al., 2019). Indeed, as proposed by the Cultural Determinants of Help Seeking model, disclosure of a suffering experience and help seeking process are more likely to happen if distress perceptions are recognized and labeled as significant and abnormal (Saint Arnault, 2009). On the contrary, normalization hinders the recognition of the abuse as a problem because violence is depicted as a normal part of a relationship (Morrison 
et al., 2006), or because women do not label their experience as abuse unless severe injuries are involved (Lundgren, 2004; McLeod et al., 2010). Based on this review, we define the normalization of GBV against women as all of those globally widespread cultural beliefs and values that sustain and justify GBV perpetration by presenting it as a normal component of male-female relationships.

Surprisingly, although many studies about violence against women generically refer to the concept of normalization as one of the critical factors involved in maintaining women in a subordinated position to men, there is a lack of instruments addressing the normalization of GBV against women and its constitutive sociocultural dimensions. Acknowledging this significant gap in scientific literature, the purpose of this study was to develop a valid and reliable measure for the assessment of the normalization of GBV against women and provide the scientific community a culturally relevant instrument that could be used to investigate this phenomenon cross-culturally.

An international and multicultural team developed the NGBV scale to account for the various cultural beliefs and values that sustain and justify violence against women in different cultural and social contexts. The relevance of multicultural measures has been stressed by previous research suggesting the need to assess the cultural validity of quantitative instruments used for cross-cultural research (see Harkness et al., 2010). This paper describes the development of a research tool that measures internalized GBV's normalization for women who have experienced abuse. We developed this instrument by conducting a large mixed-methods study. This paper reports the qualitative portion of the study (relying on expert panels, analysis of in-depth interviews, and cognitive interviewing) that allowed us to develop the instrument and assess its content and face validity. Through this paper, we focus on describing the procedures used to craft the scale by our multinational research team, a process that, despite its importance, is rarely reported in methodological literature (Boateng et al., 2018). In other studies, the quantitative portion of the mixed method study, examining the scale's psychometric properties, are reported.

\section{Methods}

Following the methodological best-practices for scale development in social research highlighted by Boateng and colleagues (2018), we developed the NGBV scale following a 3-phase process, consisting of (1) identifying the domain to be addressed by the scale; (2) generating the items; and (3) assessing the content validity of the scale. Figure 1 illustrates the activities in each phase realized by our research team to develop the NGBV scale.

\section{Phase 1: Identification of the Domain}

\section{Expert Panel Workshop}

A multicultural and multidisciplinary expert panel workshop was organized to identify the primary categories of beliefs and values that normalize GBV in various 


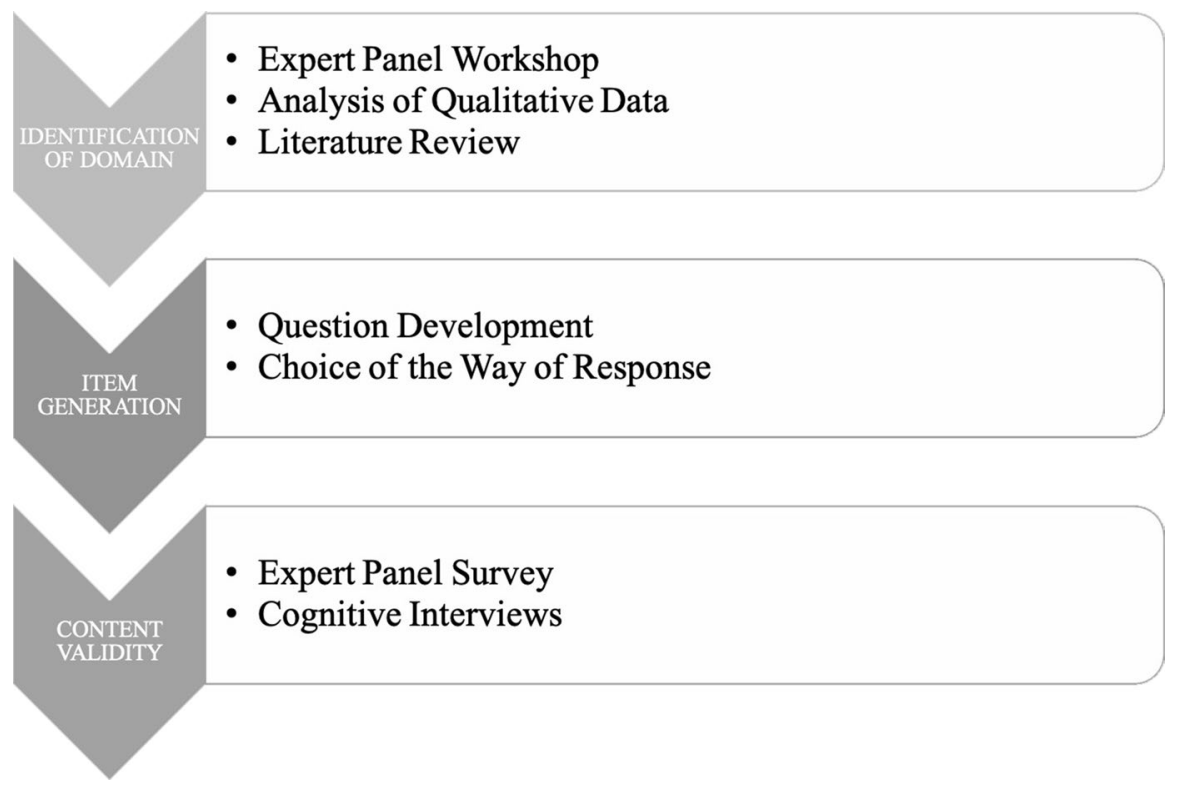

Fig. 1 Overview of the three phases of the NGBV scale development

social and cultural contexts. Twenty-three experts participated in the workshop, from Finland (1), Greece (4), Iceland (3), Ireland (2), Israel (2), Italy (2), Japan (1), Palestine (1), Romania (1), Turkey (1), South Korea (1) and USA (4) respectively. ${ }^{1}$ Members of the workshop were asked to do prework in which they gathered with colleagues in their home countries to generate lists of beliefs, values, and behaviors that normalized violence in their culture. In the workshop, international groups of four people worked together to compare their lists and discuss whether the items were culturally unique or shared among the cultures. After completing this task, each group wrote their normalization concepts on post notes and placed them on a wall. Next, all the members of the workshop worked together to group these concepts into dimensional categories.

\section{GBV Survivors' Interview Analysis}

Using the dimensions generated by the multicultural expert panel, we examined the normalization codes in previously analyzed qualitative datasets. This analytic extraction allowed us to investigate the dimensions of normalization from the point of view of GBV survivors. The qualitative datasets consisted of 70 interviews following the

\footnotetext{
${ }_{1}$ Experts participating in the workshop are scholars and professionals who are members of the MiStory network (https://mistory-traumarecovery.org/), a research collaborative working around the world to use safe and trauma-informed methods that illuminate the interactions among cultural context, the self, gender, and trauma recovery.
} 
Clinical Ethnographic Narrative Interview protocol (CENI; Saint Arnault, 2017). The dataset was created by merging smaller datasets from four studies that used the same research and analytic design to understand the GBV traumatic experience in different survivor samples. All the data in these datasets had already been thematically coded (Braun \& Clarke, 2006) for other research questions, and all the code lists contained normalization of GBV codes. For this purpose, we extracted these coded quotations to conduct another round of thematic analysis to clarify, inform, and instantiate the NGBV dimensions developed by the expert panel in the workshop. The interview data used for code extraction included 24 sexual assault US survivors (Sinko et al., 2020), 24 US GBV survivors (Sinko \& Saint Arnault, 2020), 12 Irish domestic abuse survivors (Sinko et al., 2019), and 10 Greek GBV survivors (Unpublished raw data).

\section{Review of Existing Scales}

We reviewed the literature regarding similar measurement instruments to define and refine the dimensions of NGBV generated in the expert panel and from the qualitative datasets. Because, to our knowledge, there was not an existing instrument measuring the normalization of GBV, we reviewed several available tools measuring beliefs, values, or attitudes towards acceptance of GBV (Perrin et al., 2019), rape (Burt, 1980), domestic violence (Peters, 2008; Şahin \& Dişsiz, 2009; Yount et al., 2014) or sexism (Benson \& Vincent, 1980; García-Cueto et al., 2015; Glick \& Fiske, 1996). Most of these were developed and validated with the general population in various countries.

\section{Phase 2: Item Generation}

In phase 2, an initial pool of 26 items was developed in the English language. The quotations from our empirical interview data extraction helped us refine the wording for the items. A 5-point Likert scale was selected to assess the respondent's level of agreement for each item. According to some research reports indicating that negatively worded items can negatively affect research surveys (Barnette, 2000; Dalal \& Carter, 2014), especially in cross-cultural studies (Wong et al., 2003), we decided not to create reverse items in our scale.

\section{Phase 3: Content and Face Validity Assessment}

To ensure that the scale's items were properly capturing the dimensions we intended to measure, we assessed the content and face validity of the NGBV scale using a two-step process, combining evaluation by expert judges and cognitive interviewing, as suggested in methodological literature (Boateng et al., 2018; DeVellis, 2016; McPhail, 2007). The subsequent phase, including psychometric evaluation and refinement of the tool, is currently being conducted. 


\section{Expert Judges' Evaluation}

The initial 26-item version of the NGBV scale was independently evaluated by ten experts representing nine cultural perspectives (Greek, US, Jewish, Turkish, Italian, Finnish, Romanian, South Korean, and Icelandic) to improve the content validity of the scale from a multicultural perspective. The experts were asked to rate each item's relevance and clarity on a 4-point Likert scale and propose revisions to clarify items.

\section{Cognitive Interviewing}

To assess the face validity of the NGBV scale, as a relevant component of content validity (Haynes, 1995), we used cognitive interviewing (CI). CI is a method for empirically studying how participants mentally process and respond to survey questionnaires. When used for pretesting, it consists of administering a draft version of an instrument to end-user populations, asking them to verbalize their thoughts while filling it out (Willis, 2009). In our study, the CI step's primary purposes were to ensure that the scale's items were meaningful and understandable to the target population, and to involve them in developing the scale through their observations. Moreover, since we were conducting interviews in multiple countries, our procedures and analysis were guided by recommendations for cross-cultural CI (Willis, 2015; Willis \& Miller, 2011), which is a variant of standard cognitive testing that has a greater focus on sociocultural factors that influence the survey-response process. This approach is increasingly being used to establish the cross-cultural equivalence of instruments (Willis, 2015).

Before conducting the interviews, the scale was translated from English into four languages (Greek, Icelandic, Italian, and Turkish) using a translation and backtranslation procedure (Brislin, 1970). We interviewed 31 women from five different countries, with approximately half of each country's participants self-identifying as GBV survivors (see Table 1). In each country, one research team member conducted all the interviews. The interviewers were native speakers of the language under study at their site, and all were fluent in English. The interviews took place between November 2019 and January 2020, in a location chosen by participants.

A combination of the think-aloud technique and verbal probing, as has been recommended for cross-cultural CI (Willis, 2015), was used to elicit participants' understanding and interpretation of the scale items. The probing was done retrospectively, relying on both structured and flexible probes. Written notes were taken by the interviewers during and after the interviews, documenting each participant's thoughts and responses. We decided not to audio or video-record CI to protect participants' anonymity and privacy and prevent the discomfort that could have been caused by the awareness of being recorded while talking about a sensitive topic. While we faced the consequent risk of data loss, we provided all interviewers a standard summary template to keep systematic notes about the interviews' data. After all the interviews were conducted, the analytic process included a successive aggregation technique (Willis \& Miller, 2011), in which interviewers used the standard summary template to summarize their data on an item-by-item basis. 
Table 1 Socio-demographics information about the CI participants

$\begin{array}{ll}\text { Participants }(\mathrm{N}=31) & \text { GBV survivors }(n=17) \\ \text { Age } & \text { General population }(n=14) \\ & \text { Range 20-68 years } \\ \text { Country } & \text { (mean age }=44.5 \text { years) } \\ & \text { Greece }(n=6) \\ & \text { Iceland }(n=6) \\ & \text { Italy }(n=6) \\ & \text { Turkey }(n=4) \\ \text { Level of education } & \text { USA }(n=9) \\ & \text { Primary school }(n=2) \\ & \text { Intermediate school }(n=2) \\ \text { Motherhood } & \text { High school diploma }(n=8) \\ & \text { University degree }(n=19) \\ & \text { Participants who had children }(n=20)\end{array}$

These interview summaries were the primary source for the joint analysis process. Next, the international team held a collaborative analysis meeting. The goals of this meeting were to analyze and synthesize the data and produce a single set of observations for each item across the countries. This team meeting consisted of gathering the suggested revisions from the participants, and identification of unclear concepts or phrasing. Finally, we examined the items for shared relevance and general cultural attunement of the items. Similar insights about the scale were identified across all the research sites so no further interviews were needed.

\section{Findings}

\section{Identification of the Domain}

In phase 1, we defined the domain and its constitutive dimensions. We began by conducting an expert panel workshop to identify the major dimensions that support the normalization of GBV's domain in various social and cultural contexts. These included cultural beliefs about women's responsibility for men's behavior, internalized differentials in male-female relationships, cultural beliefs about women's and men's presumed biology, cultural expectations about women's orientation toward family, the conceptualization of love, and beliefs about women's strength. Table 2 provides a brief overview of the identified dimensions and data sources.

Next, the extraction of the normalization quotations from previously analyzed qualitative data yielded an additional two dimensions. These included the expectation that "the house is the primary and proper women's domain" and the attitude that the use of violence against women is a natural part of women's daily lives across multiple life contexts outside of the relationship sphere. 


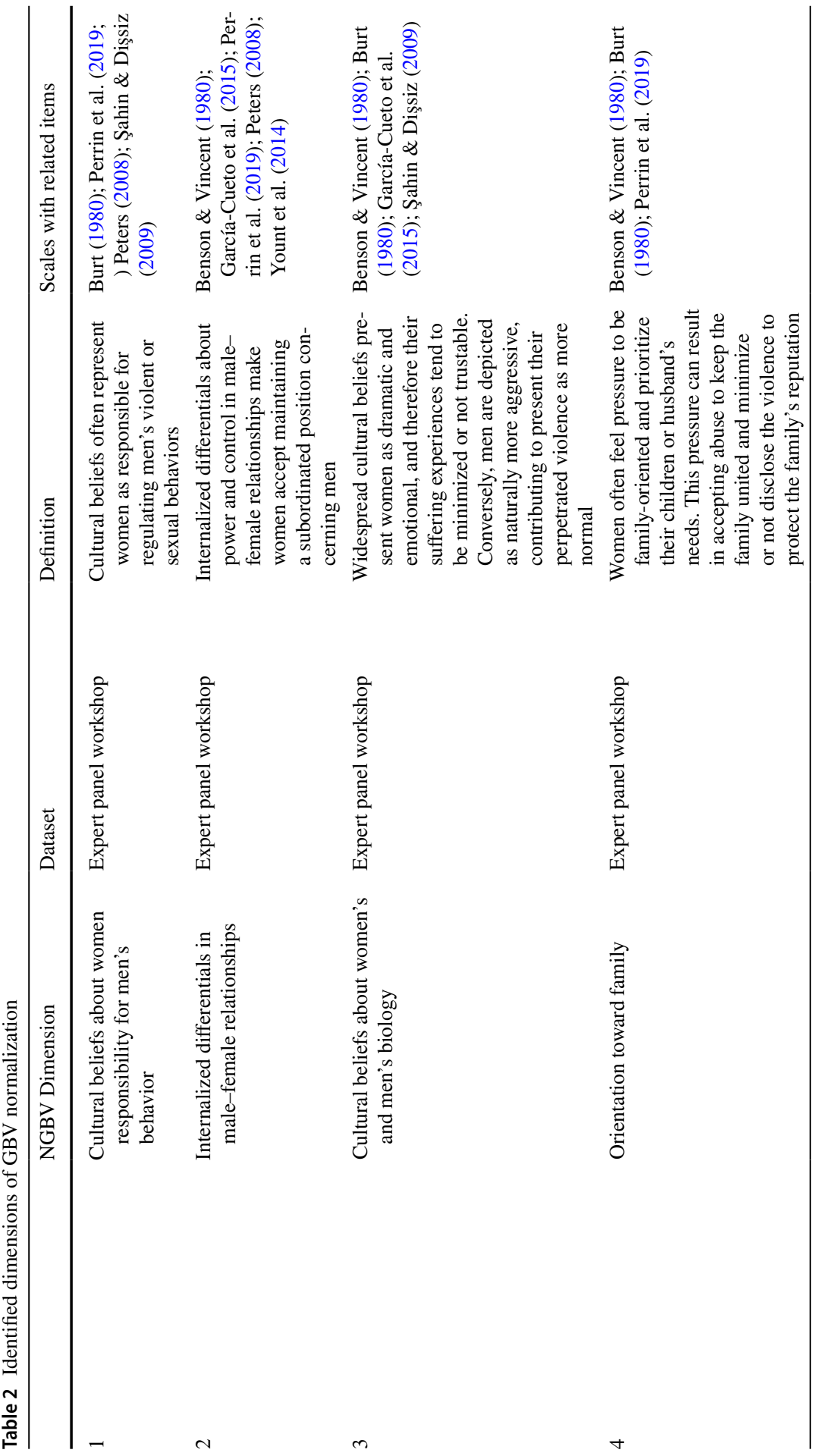




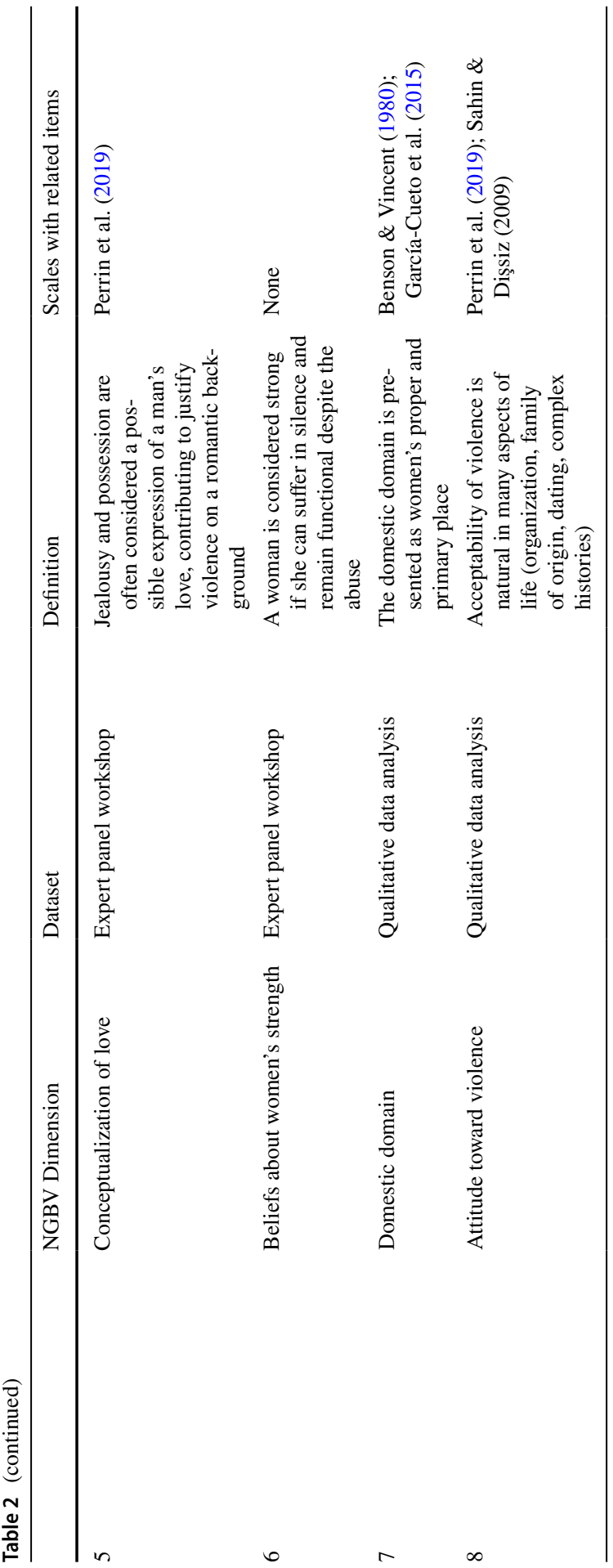


Finally, our review of existing scales measuring beliefs, values, or attitudes towards acceptance of GBV helped us find some overlaps between the normalization dimensions we identified in the expert panel workshop and the qualitative data analysis quotation extractions, as shown in Table 2. We found that other instruments partially assess cultural beliefs about women responsibility on men's behaviors, internalized differentials in male-female relationships, cultural beliefs about men and women's "biology," orientations towards family and the domestic domain, conceptualizations about love, and attitudes toward violence against women. However, the dimensions related to beliefs about women's strength and concepts about reasons to stay silent seemed to be heretofore unexplored topics in these instruments. Moreover, we ascertained that it would require many of these scales to assess all GBV normalization dimensions fully.

\section{Content Validity Assessment}

Results of the expert judges' evaluation are presented in Table 3. Ratings and comments were used to refine the items. In general, all the items were deemed relevant. We used the comments and revision suggestions to condense redundant items, add new items, and reword several items. The 26 original proposed items were thus reduced to 21 items (see Table 4 ).

The cognitive interview results suggested that participants thought that the wording, response option, and layout of the 21-item scale were clear. The cognitive interviews also confirmed that they grasped the items' intended meaning. Although some cultural nuances were uncovered (noted in Table 4), issues regarding the same items emerged repeatedly. Participants thought that most of the items' relevance and appropriateness reflected traditional and stereotyped gender roles that did not capture their own beliefs, especially for the younger respondents who talked about some items sounding "outdated." Much of the discussion about the instrument centered on how cultural beliefs about gender roles had changed over time or were perhaps generational, suggesting that the scale was probably more descriptive of the older generations' attitudes. However, participants also described a state of inner conflict about their answer to many items, feeling the need to answer both what they think and what the society presents to them as "normal." These response patterns were described of as resistance to the ideas in society, and women often said that these should not be present in an ideal world. However, participants acknowledged that these beliefs were still relevant in modern society, even for younger women.

Regardless of the participants' age, we found that GBV survivors were more likely to believe (or had believed) many of the statements at some point in their lives compared with those who had not had GBV experiences. The survivors also reported having changed their beliefs as a result of leaving the violence. Indeed, although there was a general resistance to the idea that victims of abuse would ever believe these things, most of the CI survivor respondents had an awakening consciousness that they had believed these things in the past within the interview itself.

Participants' comments and suggestions helped us refine the instrument by merging some items, splitting one item, deleting one item, and adding two additional 


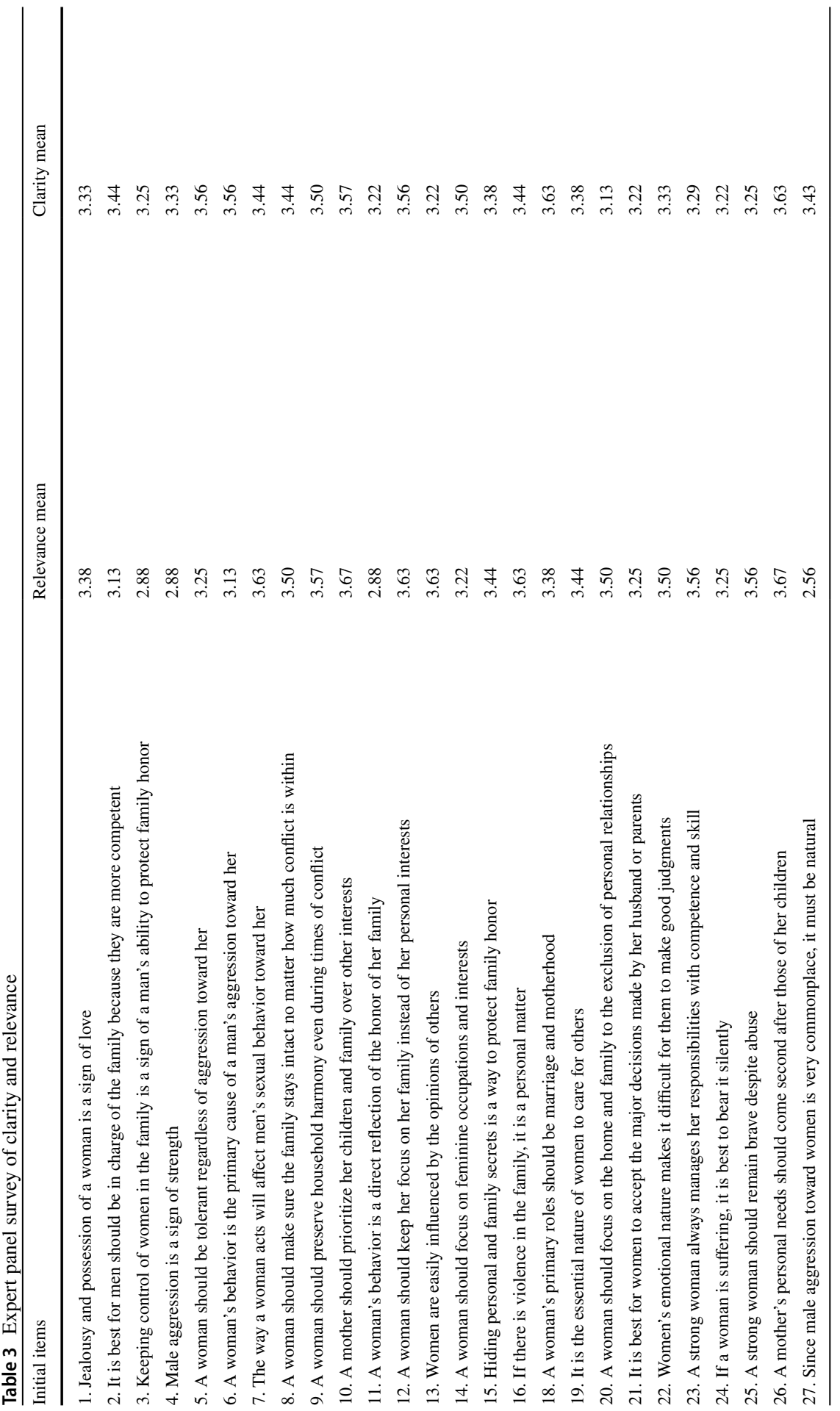




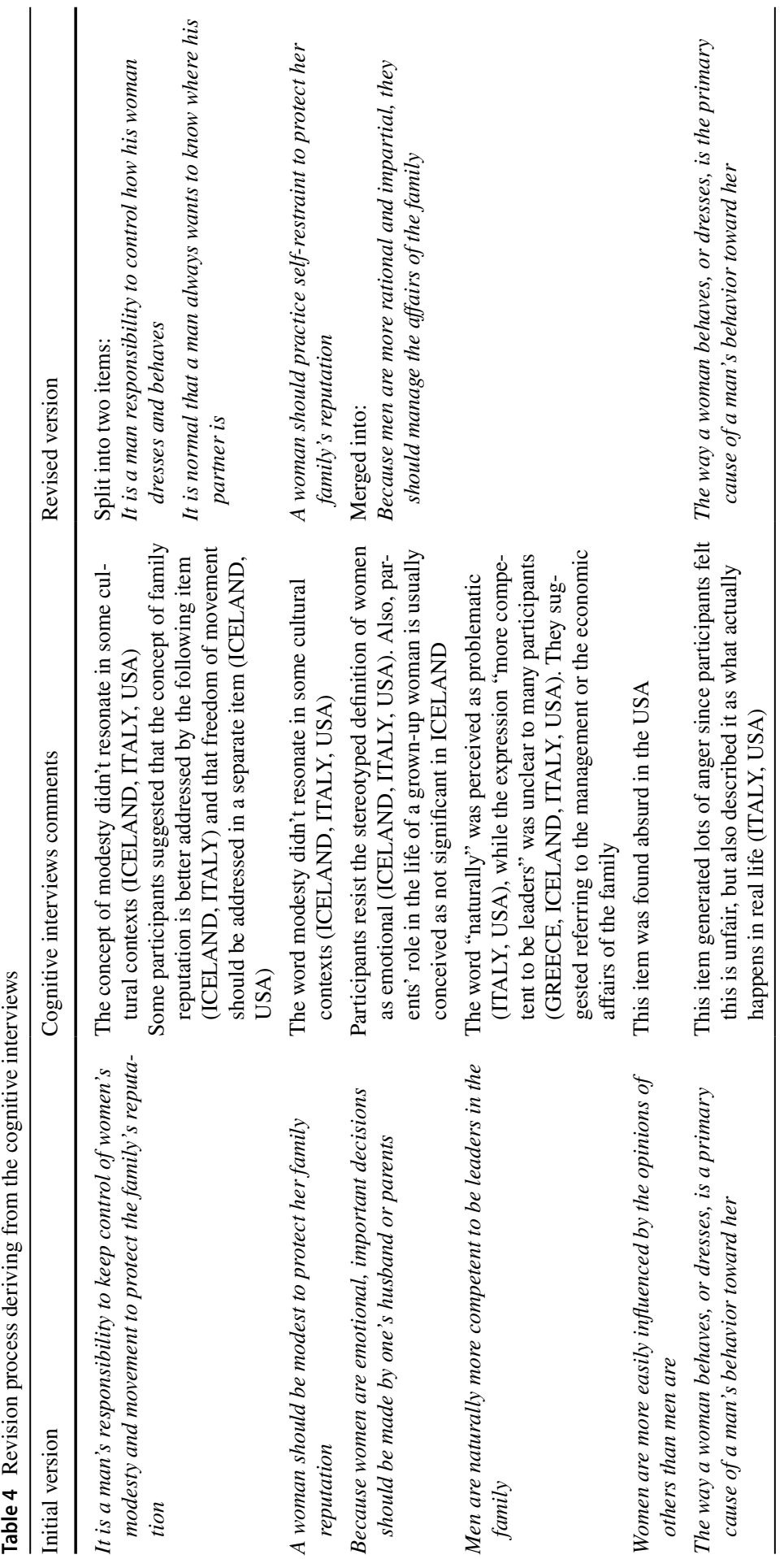




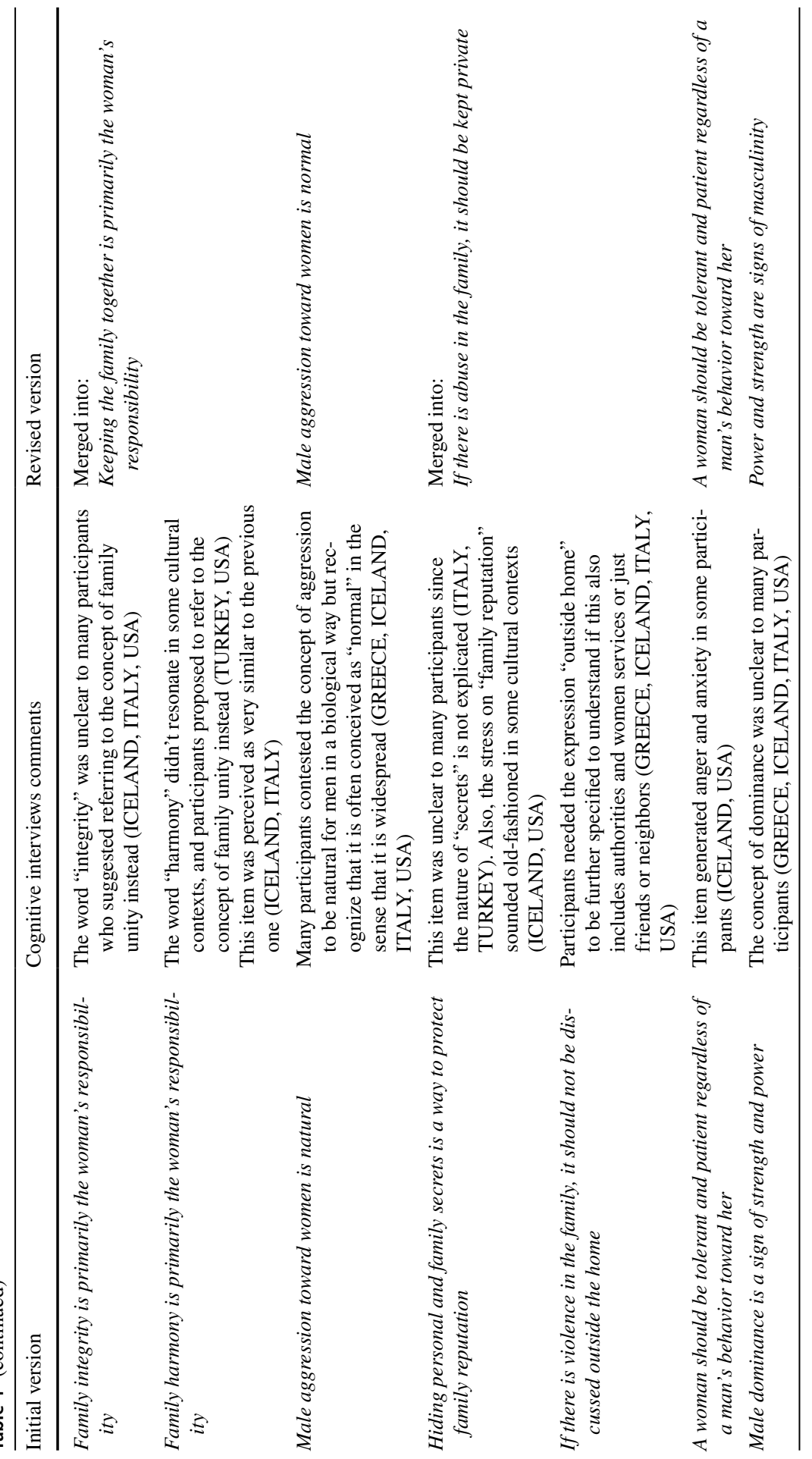




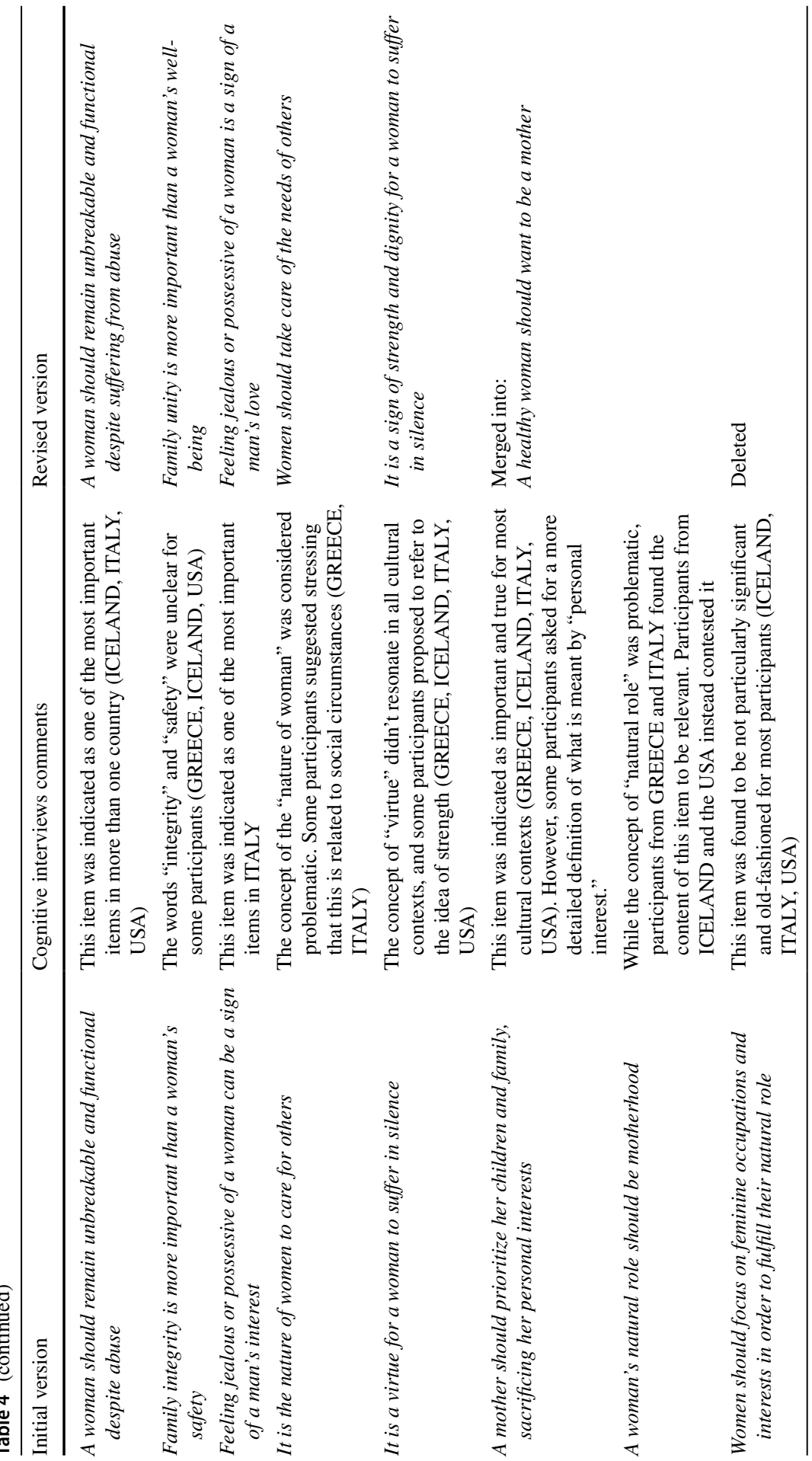




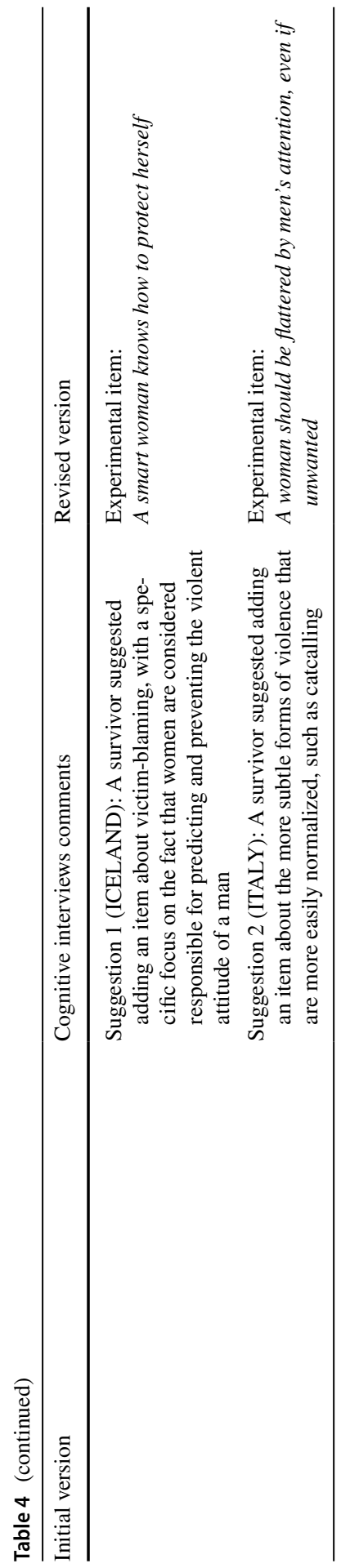


experimental items that addressed normalization dimensions that emerged in the CI interviews. Overall, the 21 items were reduced to 18 items. The interviews' findings also helped us improve our initial translations, capturing both linguistic translation and cultural nuance issues. The revision process deriving from the $\mathrm{CI}$ and the final 18 -item version of the scale are reported in Table 4.

A key overall finding was regarding the comments the participants made about how their perspectives about the normalization of GBV have evolved, both due to the healing the survivors had achieved and the evolution of social consciousness in their countries. In general, the CI respondents' comments were essentially, "I used to think that, but I don't anymore." Acknowledging this possibility that normalization beliefs are affected by different stages of a healing journey, in our final version of the instrument, we created a differential the response option of the scale by asking the respondents to give two answers for each item. The first asks how strongly they believed that statement during the times they were experiencing GBV. The second asks how much they believe that statement at the time of the scale administration (see Appendix).

\section{Discussion}

This study presents an intercultural approach for the conceptual development and initial validation of a new instrument to measure the normalization of GBV against women. The NGBV scale was developed to assess internalized normalization beliefs that may be barriers to self-disclosure and may also keep shame and self-doubt alive in the survivor. The 18-item scale measures various dimensions of the domain of interest, including cultural beliefs about women responsibility for men's behavior, internalized differentials in male-female relationships, cultural beliefs about women's and men's presumed biology, cultural expectations about women's role in the family, and the domestic sphere, conceptualizations of love, beliefs about women's strength, and general violence against women attitudes.

To our knowledge, the NGBV scale is the first instrument explicitly addressing the normalization of GBV against women that has been designed to be used with violence survivors. Moreover, the NGBV scale distinguishes itself from other existing scales that address similar topics (see, for example, Benson \& Vincent, 1980; Burt, 1980; García-Cueto et al., 2015; Glick \& Fiske, 1996; Perrin et al., 2019; Peters, 2008; Şahin \& Dişsiz, 2009; Yount et al., 2014) because it considers unexplored dimensions of NGBV, such as beliefs about strength and possible reasons for staying silent about abuse. Finally, another unique feature of the NGBV scale derives from the fact that it has been developed by a multicultural team using techniques aimed to capture cultural aspects of normalization that might be shared across women from diverse groups.

Our CI participants often contested the idea that normalization of GBV was still relevant in their culture, describing it as the product of "old-fashioned beliefs" that no longer affected their social environment. Conversely, their state of inner conflict while answering many items-leading us to change the scale's response optionsuggested that these beliefs were still relevant in modern society. This contradiction 
hints that patriarchal norms and gender stereotypes supporting gendered violence can be internalized by women (see also McCarry \& Lombard, 2016). Moreover, it suggests the implicit nature of culture, which operates within individuals by encompassing systems of meanings and rules and embodied tendencies that organize how people understand themselves and their world and how they behave within their social world (Luria, 1976). Thus, we can hypothesize that GBV survivors may not explicitly recognize how these internalized sociocultural norms and values impact their engagement in recovery from GBV, making this scale a valuable tool in our quest to uncover the implications of these culturally held beliefs and help seeking. Because NGBV is at least partially implicit, this NGBV scale may be a helpful tool to assess and perhaps intervene to de-normalize NGBV beliefs for survivors. For example, we found emerging consciousness about the prevelence of these beliefs in sociaty and in themselves even as the participants took the scale. However, these interventions, similar to other implicit bias interventions, must focus on non-conscious beliefs and explicit ones.

A relevant aspect of the NGBV scale development process that requires further reflection and discussion concerns the choice to change the response option of the scale, providing for the possibility to differentiate the current beliefs from the beliefs when the violence occurred. We used this strategy to reduce respondents' abovementioned inner conflict by allowing them to express the perceived changes in their beliefs through time. Therefore, changing the response option has the advantages of reducing the participants' distress while answering the items and account for a subjective differential between the present and the time of the violence. However, this response option may encourage participants to exaggerate the difference between the present and the past. For this reason, the psychometric study that is being conducted to test the reliability and validity of this scale in multiple cultural contexts includes a test-retest measure aimed at investigating if responses are reliable using this double response-option or if the scale is better to be used only referencing to the present moment. However, the initial validity assessment conducted in the present study suggests that this scale has good content and face validity, and the differential response option comes directly from the participants.

Given this instrument's multicultural nature, we encourage professionals to use the NGBV scale to evaluate interventions or programs' effectiveness to decrease GBV or to de-normalizing such violence. International use can help researchers develop a clearer understanding of cultural and social norms that sustain violence acceptance and normalization.

\section{Limitations and future studies}

We recognize some limitations of this study, including that only one round of CI was conducted in each country. Having two or three testing rounds, iterative in nature, would have been ideal. Additionally, the use of written notes alone in the CI data collection phase, with no audio or video recording, might have resulted in some data loss. However, the data collection was not stopped until intra-site saturation was reached, and when inter-site confirmation was achieved. Moreover, several 
issues had been identified and adequately addressed. Another limitation is that, in some countries, the cognitive interview participants were more highly educated than the general population in the participating countries. Nonetheless, our triangulated approach helped to ensure the face validity of the items. For future studies, we recommend other researchers use the NGBV scale in different cultural settings to assess its validity and the generalizability of its results in different samples of participants with diverse SES, and especially with younger participants than those involved in the present study.

This scale was developed to assess internalized normalization beliefs that may prevent GBV experience and help seeking disclosure. However, these normalization dimensions are critical to assess in other populations as well, such as in prevention research or evaluation of de-normalization efforts. Therefore, we believe that a possible clinical application for this scale could be related to the study of the healing process of de-normalization in the future. Finally, although the scale was designed for use with trauma survivors who are predominately women, the assessed dimensions may also relate to other survivors of GBV, such as sexual minorities or those with gender-nonconforming identities. Therefore, future research could use the NGBV scale to assess the normalization of violence in these populations.

\section{Appendix: Final form of the NGBV Scale}

See Table 5 . 


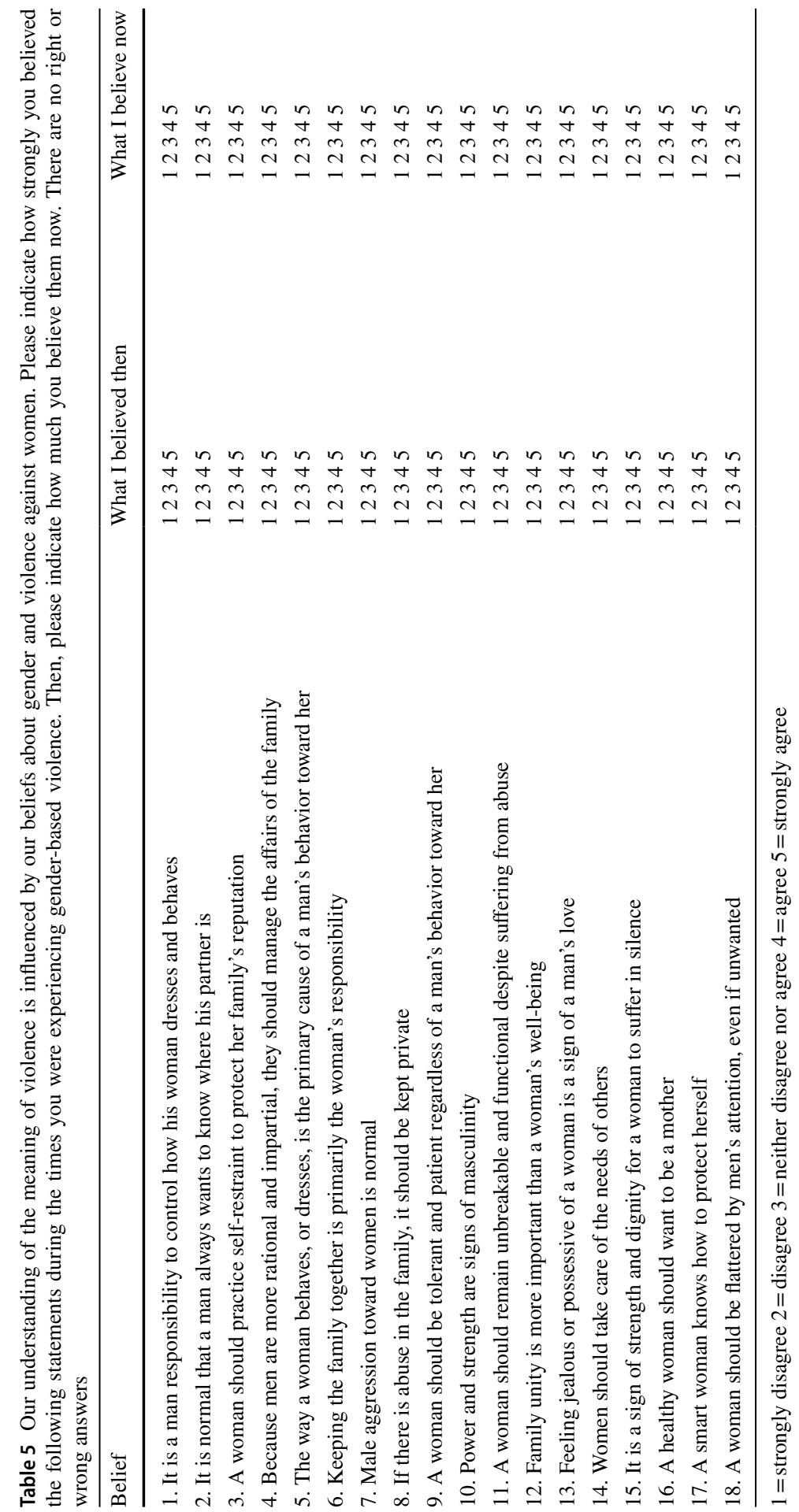


Funding Open access funding provided by Università degli Studi di Padova within the CRUI-CARE Agreement. No funding was received for conducting this study.

Data Availability Data and materials needed to reproduce all of the reported results are available on request from the senior author.

\section{Declarations}

Conflict of interest The authors have no conflicts of interest to declare that are relevant to the content of this article.

Consent to participate Informed consent was obtained from all individual participants included in the study.

Ethical Approval Approval was obtained from the ethics committee of Michigan University (IRB HUM00168381 and HUM00091662). The procedures used in this study adhere to the tenets of the Declaration of Helsinki.

Open Access This article is licensed under a Creative Commons Attribution 4.0 International License, which permits use, sharing, adaptation, distribution and reproduction in any medium or format, as long as you give appropriate credit to the original author(s) and the source, provide a link to the Creative Commons licence, and indicate if changes were made. The images or other third party material in this article are included in the article's Creative Commons licence, unless indicated otherwise in a credit line to the material. If material is not included in the article's Creative Commons licence and your intended use is not permitted by statutory regulation or exceeds the permitted use, you will need to obtain permission directly from the copyright holder. To view a copy of this licence, visit http://creativecommons.org/licen ses/by/4.0/.

\section{References}

Barnette, J. J. (2000). Effects of stem and Likert response option reversals on survey internal consistency: If you feel the need, there is a better alternative to using those negatively worded stems. Educational and Psychological Measurement, 60(3), 361-370. https://doi.org/10.1177/00131640021970592

Benson, P. L., \& Vincent, S. (1980). Development and validation of the sexist attitudes toward women scale (SATWS). Psychology of Women Quarterly, 5(2), 276-291. https://doi.org/10.1111/j.14716402.1980.tb00962.x

Bloom, S. S. (2008). Violence against women and girls: A compendium of monitoring and evaluation indicators. Chapel Hill.

Boateng, G. O., Neilands, T. B., Frongillo, E. A., Melgar-Quiñonez, H. R., \& Young, S. L. (2018). Best practices for developing and validating scales for health, social, and behavioral research: a primer. Frontiers in Public Health, 6, 149. https://doi.org/10.3389/fpubh.2018.00149

Braun, V., \& Clarke, V. (2006). Using thematic analysis in psychology. Qualitative Research in Psychology, 3(2), 77-101. https://doi.org/10.1191/1478088706qp063oa

Brislin, R. W. (1970). Back-translation for cross-cultural research. Journal of Cross-Cultural Psychology, 1(3), 185-216. https://doi.org/10.1177/135910457000100301

Burt, M. R. (1980). Cultural myths and supports for rape. Journal of Personality and Social Psychology, 38(2), 217-230. https://doi.org/10.1037/0022-3514.38.2.217

Butler, J. (1999). Gender trouble. Routledge.

Dalal, D. K., \& Carter, N. T. (2014). Negatively worded items negatively impact survey research. In C. E. Lance \& R. J. Vandenberg (Eds.), More statistical and methodological myths and urban legends: doctrine, verity and fable in organizational and social sciences (pp. 112-132). Routledge.

DeVellis, R. F. (2016). Scale development: Theory and applications. Sage publications.

Foucault, M. (1990). The history of sexuality. Gallimard. 
French, S. L. (2003). Reflections on healing: Framing strategies utilized by acquaintance rape survivors. Journal of Applied Communication Research, 31(4), 298-319. https://doi.org/10.1080/1369681032 000132573

Fugate, M., Landis, L., Riordan, K., Naureckas, S., \& Engel, B. (2005). Barriers to domestic violence help seeking: Implications for intervention. Violence against Women, 11, 290-310. https://doi.org/ 10.1177/1077801204271959

García-Cueto, E., Rodríguez-Díaz, F. J., Bringas-Molleda, C., López-Cepero, J., Paíno-Quesada, S., \& Rodríguez-Franco, L. (2015). Development of the gender role attitudes scale (GRAS) amongst young Spanish people. International Journal of Clinical and Health Psychology, 15(1), 61-68. https://doi.org/10.1016/j.ijchp.2014.10.004

Glick, P., \& Fiske, S. T. (1996). The ambivalent sexism inventory: Differentiating hostile and benevolent sexism. Journal of Personality and Social Psychology, 70(3), 491-512. https://doi.org/10.1037/ 0022-3514.70.3.491

Gracia, E. (2004). Unreported cases of domestic violence against women: towards an epidemiology of social silence, tolerance, and inhibition. Journal of Epidemiology and Community Health, 58(7), 536-537. https://doi.org/10.1136/jech.2003.019604

Harkness, J. A., Braun, M., Edwards, B., Johnson, T. P., Lyberg, L., Mohler, P. P., Pennell, B.-E., \& Smith, T. W. (Eds.), (2010). Wiley series in survey methodology. Survey methods in multinational, multiregional, and multicultural contexts. John Wiley \& Sons, Inc. https://doi.org/10.1002/97804 70609927

Haynes, S. N., Richard, D., \& Kubany, E. S. (1995). Content validity in psychological assessment: A functional approach to concepts and methods. Psychological Assessment, 7(3), 238-247. https://doi. org/10.1037/1040-3590.7.3.238

Lundgren, E. (2004). The process of normalising violence. National Organization of Women's Shelters and Young Women's Shelters in Sweden.

Luria, A. R. (1976). Cognitive development: Its cultural and social foundations. Harvard University Press.

McCarry, M., \& Lombard, N. (2016). Same old story? Children and young people's continued normalisation of men's violence against women. Feminist Review, 112(1), 128-143.

McLeod, A. L., Hays, D. G., \& Chang, C. Y. (2010). Female intimate partner violence survivors' experiences with accessing resources. Journal of Counseling \& Development, 88(3), 303-310. https://doi. org/10.1002/j.1556-6678.2010.tb00026.x

McPhail, S. M. (2007). Alternative validation strategies: Developing new and leveraging existing validity evidence. Wiley.

Messerschmidt, J. (2012). Gender, heterosexuality, and youth violence: The struggle for recognition. Rowman \& Littlefield.

Morrison, K. E., Luchok, K. J., Richter, D. L., \& Parra-Medina, D. (2006). Factors influencing help-seeking from informal networks among African American victims of intimate partner violence. Journal of Interpersonal Violence, 21(11), 1493-1511. https://doi.org/10.1177/0886260506293484

Overstreet, N. M., \& Quinn, D. M. (2013). The intimate partner violence stigmatization model and barriers to help seeking. Basic and Applied Social Psychology, 35(1), 109-122. https://doi.org/10.1080/ 01973533.2012 .746599

Perrin, N., Marsh, M., Clough, A., Desgroppes, A., Phanuel, C. Y., Abdi, A., Kaburu, F., Heitmann, S., Yamashina, M., Ross, B., Read-Hamilton, S., Turner, R., Heise, L., \& Glass, N. (2019). Social norms and beliefs about gender-based violence scale: a measure for use with gender-based violence prevention programs in low-resource and humanitarian settings. Conflict and Health, 13(1), 6. https://doi.org/10.1186/s13031-019-0189-X

Peters, J. (2008). Measuring myths about domestic violence: Development and initial validation of the domestic violence myth acceptance scale. Journal of Aggression, Maltreatment \& Trauma, 16(1), 1-21. https://doi.org/10.1080/10926770801917780

World Health Organization. (2016). Responding to intimate partner violence and sexual violence against women: WHO clinical and policy guidelines.

Şahin, N. H., \& Dişsiz, M. (2009). Development study of attitudes towards domestic violence scale in healthcare workers. Journal of Human Sciences, 6(2), 263-274.

Saint Arnault, D. (2009). Cultural determinants of help seeking: A model for research and practice. Research and Theory for Nursing Practice, 23(4), 259-278. https://doi.org/10.1891/1541-6577. 23.4.259 
Saint Arnault, D. M. (2017). The use of the clinical ethnographic narrative interview to understand and support help seeking after gender-based violence. TPM Testing, Psychometrics, Methodology in Applied Psychology, 24(3), 423-436. https://doi.org/10.4473/TPM24.3.8

Sinko, L., Burns, C. J., O’ Halloran, S., \& SaintArnault, D. (2019). Trauma recovery is cultural: understanding shared and different healing themes in Irish and American survivors of gender-based violence. Advance Online Publication, 21, 1-2. https://doi.org/10.1177/0886260519829284

Sinko, L., Munro-Kramer, M., Conley, T., \& Saint Arnault, D. (2020). Internalized messages: The role of sexual violence normalization on meaning-making after campus sexual violence. Journal of Aggression, Maltreatment \& Trauma. https://doi.org/10.1080/10926771.2020.1796872

Sinko, L., \& Saint Arnault, D. (2020). Finding the strength to heal: understanding recovery after genderbased violence. Violence against Women, 26(12-13), 1616-1635. https://doi.org/10.1177/10778 01219885185

United Nations. (1993). Declaration on the elimination of violence against women. United Nations Department of Public Information.

Willis, G. B. (2009). Cognitive interviewing. In P. J. Lavrakas (Ed.), Encyclopedia of survey research methods (pp. 106-109). SAGE.

Willis, G. B. (2015). The practice of cross-cultural cognitive interviewing. Public Opinion Quarterly, 79(S1), 359-395. https://doi.org/10.1093/poq/nfu092

Willis, G. B., \& Miller, K. (2011). Cross-cultural cognitive interviewing: Seeking comparability and enhancing understanding. Field Methods, 23(4), 331-341. https://doi.org/10.1177/1525822X11 416092

Wirtz, A. L., Poteat, T. C., Malik, M., \& Glass, N. (2020). Gender-based violence against transgender people in the United States: A call for research and programming. Trauma, Violence, \& Abuse, 21(2), 227-241. https://doi.org/10.1177/1524838018757749

Wong, N., Rindfleisch, A., \& Burroughs, J. E. (2003). Do reverse-worded items confound measures in cross-cultural consumer research? The case of the material values scale. Journal of Consumer Research, 30(1), 72-91. https://doi.org/10.1086/374697

Wood, J. T. (2001). The normalization of violence in heterosexual romantic relationships: Women's narratives of love and violence. Journal of Social and Personal Relationships, 18(2), 239-261. https:// doi.org/10.1177/0265407501182005

Yount, K. M., VanderEnde, K., Zureick-Brown, S., Anh, H. T., Schuler, S. R., \& Minh, T. H. (2014). Measuring attitudes about intimate partner violence against women: The ATT-IPV Scale. Demography, 51(4), 1551-1572. https://doi.org/10.1007/s13524-014-0297-6

Publisher's Note Springer Nature remains neutral with regard to jurisdictional claims in published maps and institutional affiliations.

\section{Authors and Affiliations}

\section{Maddalena Rodelli ${ }^{1}$ (D) Kleio Koutra ${ }^{2}$ (D) Karen Birna Thorvaldsdottir ${ }^{3}$ (D) Hulya Bilgin ${ }^{4}$ (D) Nikoleta Ratsika ${ }^{2}$ - Ines Testoni ${ }^{1}$ (D) Denise M. Saint Arnault ${ }^{5}$}

1 Department of Philosophy, Sociology, Education and Applied Psychology, University of Padova, Via Venezia, 35123 Padova, Italy

2 Department of Social Work, Institute of Agri-Food and Life Sciences, University Research Centre, Hellenic Mediterranean University, Iraklio, Greece

3 School of Health Sciences, University of Akureyri, Akureyri, Iceland

4 Department of Nursing, Univeristy of Istanbul, İstanbul, Turkey

5 Department of Health Behavior and Biological Sciences, University of Michigan, Ann Arbor, USA 